
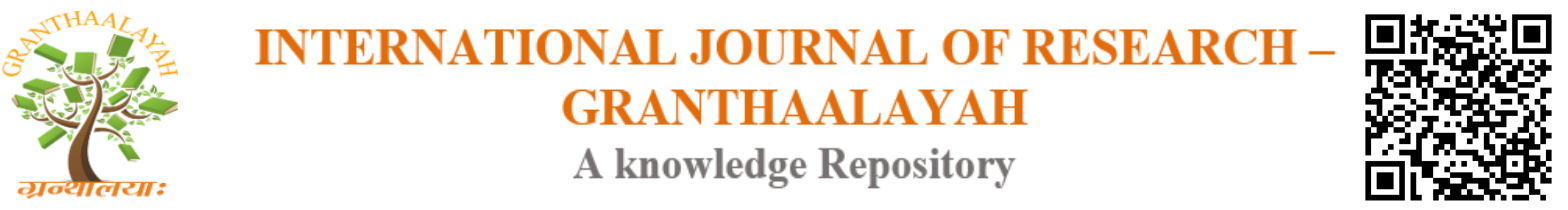

Science

\title{
SOLVING SYSTEMS OF FRACTIONAL PARTIAL DIFFERENTIAL EQUATIONS VIA TWO DIMENSIONAL LAPLACE TRANSFORMS
}

\author{
A. Aghili ${ }^{* 1}$, M.R. Masomi ${ }^{2}$ \\ ${ }^{* 1,2}$ Department of Applied Mathematics, Faculty of Mathematical Sciences, University of \\ Guilan, P.O. Box, 1841, Rasht-Iran
}

\begin{abstract}
In this article, the authors used two dimensional Laplace transform to solve non - homogeneous sub - ballistic fractional PDE and homogeneous systems of time fractional heat equations. Constructive examples are also provided.

Mathematics Subject Classification 2010: 26A33; 34A08; 34K37; 35R11; 44A10.

Keywords: Fractional Partial Differential Equations; Fractional Diffusion Equations; Two Dimensional Laplace Transform; Systems of Heat Equations.

Cite This Article: A. Aghili, and M.R. Masomi. (2017). "SOLVING SYSTEMS OF FRACTIONAL PARTIAL DIFFERENTIAL EQUATIONS VIA TWO DIMENSIONAL LAPLACE TRANSFORMS." International Journal of Research - Granthaalayah, 5(12), 406-420. https://doi.org/10.29121/granthaalayah.v5.i12.2017.528.
\end{abstract}

\section{Introduction and Preliminaries}

The problem related to partial differential equation commonly can be solved by using a special integral transform, thus many authors solved the boundary value problems by using single Laplace transform. Furthermore, two dimensional Laplace transforms in the classical sense for solving linear second order partial differential equations were used by Ditkin [11], Brychkov [13]. The Laplace transform, it can be fairly said, stands first in importance among all integral transforms for which there are many specific examples in which other transforms prove more expedient. The Laplace transform is the most powerful in dealing with both initial boundary value problems and transforms [1-9]. The two dimensional Laplace transforms is a powerful tool in applied mathematics and engineering.

The fractional derivative is one of the most interdisciplinary fields of mathematics, with many applications in physics and engineering and deals with extensions of derivatives and integrals to non-integer orders. It represents a powerful tool in applied mathematics to study a myriad of problems from different fields of science and engineering, with many breakthrough results found in mathematical physics, finance, hydrology, biophysics, thermodynamics, control theory, 
statistical mechanics, astrophysics, cosmology and bioengineering [13, 14, 15, 16]. Several definitions have been proposed for a fractional derivative. We deal with Caputo fractional derivatives only. In this section, we present the definition of this derivative.

Let us take $f$ an arbitrary integrable function. By ${ }_{a} I_{t}^{\alpha} f(t)$ we denote the fractional integral of $f$ with order $\alpha>0$ on $[0, t]$ defined as follows

$$
{ }_{a} I_{t}^{\alpha} f(t)=\frac{1}{\Gamma(\alpha)} \int_{a}^{t} \frac{f(x)}{(t-x)^{1-\alpha}} d x
$$

The above integral is sometimes called the left-sided fractional integral.

For an arbitrary real number $\alpha>0(n-1<\alpha \leq n, n \in N)$ the Caputo fractional derivatives are defined as

$$
{ }_{a}^{C} D_{t}^{\alpha} f(t)={ }_{a} I_{t}^{n-\alpha} f^{(n)}(t)=\frac{1}{\Gamma(n-\alpha)} \int_{a}^{t} \frac{f^{(n)}(x)}{(t-x)^{\alpha-n+1}} d x .
$$

The Caputo fractional derivative is a regularization in the time origin for the Riemann-Liouville fractional derivative by incorporating the relevant initial conditions. The major utility of the Caputo fractional derivative is caused by the treatment of differential equations of the fractional order for physical applications, where the initial conditions are usually expressed in terms of a given function and its derivatives of integer (not fractional order), even if the governing equation is of fractional order. If care is taken, the results obtained using the Caputo formulation can be recast to the Riemann-Liouville version and vice versa.

Let $f(t)$ be a function of $t$ specified for $t>0$. Then the Laplace transform of function $f(t)$ is defined by

$$
L\{f(t)\}=\int_{0}^{\infty} e^{-s t} f(t) d t:=F(s)
$$

If $L\{f(t)\}=F(s)$, then $L^{-1}\{F(s)\}$ is given by

$$
f(t)=\frac{1}{2 \pi i} \int_{c-i \infty}^{c+i \infty} e^{t s} F(s) d s, \quad(t>0)
$$

Where $F(s)$ is analytic in the region $\operatorname{Re}(s)>c$ and $f(t)=0$ for $t<0$.

This result is called complex inversion formula. It is also known as Bromwich's integral formula. When $n-1<\alpha \leq n$, we get the following $[14,15]$

$$
L\left\{{ }_{0}^{C} D_{t}^{\alpha} f(t)\right\}=s^{\alpha} F(s)-\sum_{k=0}^{n-1} s^{\alpha-k-1} f^{(k)}(0) .
$$


Example 1.1. Let us solve the following fractional Volterra equation of convolution Type. The Laplace transform provides a useful technique for the solution of such

Equations

$$
\lambda \int_{0}^{t} I_{0}\left(2 \sqrt{\beta(\mathrm{t}-\xi)} D_{\xi}^{\alpha} \phi(\xi) d \xi=\left(\frac{t}{\beta}\right)^{\frac{v}{2}} J_{v}(2 \sqrt{\beta t}), v+\alpha>1, \quad \phi(0)=0 .\right.
$$

Solution. Upon taking the Laplace transform of the given equation, we obtain

$$
\left(\frac{\lambda e^{\frac{\beta}{s}}}{s}\right)\left(s^{\alpha} \Phi(\mathrm{s})\right)=\frac{e^{-\frac{\beta}{s}}}{s^{v+1}},
$$

Solving the above equation, leads to

$$
\Phi(s)=\frac{1}{\lambda}\left(\frac{e^{-\frac{2 \beta}{s}}}{s^{v+\alpha}}\right) .
$$

By applying the inverse Laplace transform, we get the formal solution

$$
\phi(\mathrm{t})=\frac{1}{\lambda}\left(\frac{t}{2 \beta}\right)^{\frac{v+\alpha-1}{2}} J_{v+\alpha-1}(2 \sqrt{2 \beta t}) .
$$

Lemma 1.1 Let us consider the following system of fractional singular integro - differential equations of convolution-type with the Bessel kernel:

$$
\left\{\begin{array}{l}
{ }_{x}^{c} D_{0}^{\alpha} g_{1}(x)=f_{1}(x)-\lambda \int_{0}^{x}\left(\frac{x-t}{a}\right)^{\frac{v}{2}} J_{v}(2 \sqrt{a(x-t)}) g_{2}(t) d t \\
{ }_{x}^{c} D_{0}^{\alpha} g_{2}(x)=f_{2}(x)+\lambda \int_{0}^{x}\left(\frac{x-t}{a}\right)^{\frac{v}{2}} J_{v}(2 \sqrt{a(x-t)}) g_{1}(t) d t
\end{array}\right.
$$

Where $g_{1}(0)=g_{2}(0)=0, v>-1,0<|\lambda|<1$ and $f_{1}(x), f_{2}(x)$ are known functions.

Then the above system has the following formal solutions 


$$
\begin{aligned}
& g_{1}(x)=\sum_{k=0}^{\infty}\left(-\lambda^{2}\right)^{k} \int_{0}^{x}\left(\frac{x-t}{2 a k}\right)^{k(\alpha+v+1)+\frac{\alpha}{2}} J_{2 k(\alpha+v+1)+\alpha}(2 \sqrt{2 k a(x-t)}) f_{1}^{\prime}(t) d t \\
&-\sum_{k=0}^{\infty}(-1)^{k} \lambda^{(2 k+1)} \int_{0}^{x}\left(\frac{x-t}{(2 k+1) a}\right)^{\frac{(2 k+1)}{2}(\alpha+v+1)+\frac{\alpha}{2}} J_{(2 k+1)(\alpha+v+1)+\alpha}(2 \sqrt{a(2 k+1)(x-t)}) f_{2}^{\prime}(t) d t \\
&+f_{1}(0) \sum_{k=0}^{\infty}\left(-\lambda^{2}\right)^{k}\left(\frac{x}{2 a k}\right)^{k(\alpha+v+1)+\frac{\alpha}{2}} J_{2 k(\alpha+v+1)+\alpha}(2 \sqrt{2 k a x}) \\
&-f_{2}(0) \sum_{k=0}^{\infty}(-1)^{k} \lambda^{(2 k+1)}\left(\frac{x}{(2 k+1) a}\right)^{\frac{(2 k+1)}{2}(\alpha+v+1)+\frac{\alpha}{2}} J_{(2 k+1)(\alpha+v+1)+\alpha}(2 \sqrt{a(2 k+1) x}) . \\
& g_{2}(x)=\sum_{k=0}^{\infty}(-1)^{k} \lambda^{(2 k+1)} \int_{0}^{x}\left(\frac{x-t}{(2 k+1) a}\right) \frac{(2 k+1)}{2}(\alpha+v+1)+\frac{\alpha}{2} J_{(2 k+1)(\alpha+v+1)+\alpha}(2 \sqrt{a(2 k+1)(x-t)}) f_{1}^{\prime}(t) d t \\
&+\sum_{k=0}^{\infty}\left(-\lambda^{2}\right)^{k} \int_{0}^{x}\left(\frac{x-t}{2 a k}\right)^{k(\alpha+v+1)+\frac{\alpha}{2}} J_{2 k}(\alpha+v+1)+\alpha \\
&+f_{1}(0) \sum_{k=0}^{\infty}(-1)^{k} \lambda^{(2 k+1)}\left(\frac{x}{(2 k+1) a}\right) \frac{(2 k+1)}{2}(\alpha+v+1)+\frac{\alpha}{2} J_{(2 k+1)(\alpha+v+1)+\alpha}(2 \sqrt{a(2 k+1) x}) \\
&+f_{2}(0) \sum_{k=0}^{\infty}\left(-\lambda^{2}\right)^{k}\left(\frac{x}{2 a k}\right)^{k(\alpha+v+1)+\frac{\alpha}{2}} J_{2 k(\alpha+v+1)+\alpha}(2 \sqrt{2 k a x}) \\
& f_{2}^{\prime}(t) d t \\
&
\end{aligned}
$$

Proof - In order to solve the above system, by introducing we can rewrite the above system of partial fractional integro - differential equations in the following form

$$
{ }_{x}^{c} D_{0}^{\alpha} g(x)=f(x)+\lambda i \int_{0}^{x}\left(\frac{x-t}{a}\right)^{\frac{v}{2}} J_{v}(2 \sqrt{a(x-t)}) g(t) d t
$$

Where $g(0)=0, v>-1, a>0,0<|\lambda|<1$.

By applying the Laplace transform of both sides of the above equation term - wise we obtain

$$
\begin{aligned}
& s^{\alpha} G(s)=F(s)+\lambda i \frac{\exp \left(\frac{-a}{s}\right)}{s^{v+1}} G(s) \quad \Rightarrow \\
& G(s)=\frac{1}{s^{\alpha}-\lambda i \frac{\exp \left(\frac{-a}{s}\right)}{s^{v+1}}} F(s)=\frac{1}{s^{\alpha}\left\{1-\frac{\lambda i}{s^{\alpha+v+1} \exp \left(\frac{a}{s}\right)}\right\}} F(s) \\
& =\frac{F(s)}{s^{\alpha}} \sum_{k=0}^{\infty} \frac{(\lambda i)^{k}}{s^{(\alpha+v+1) k} \exp \left(\frac{k a}{s}\right)}=s F(s) \sum_{k=0}^{\infty} \frac{(\lambda i)^{k}}{s^{(\alpha+v+1) k+\alpha+1} \exp \left(\frac{k a}{s}\right)}
\end{aligned}
$$


Now, using the fact that

$$
L^{-1}\left\{\frac{\exp \left(\frac{-a}{s}\right)}{s^{v+1}}\right\}=\left(\frac{x}{a}\right)^{\frac{v}{2}} J_{v}(2 \sqrt{a x}),(i)^{2 k}=(-1)^{k},(i)^{2 k+1}=(-1)^{k} i
$$

Upon taking the inverse Laplace transform of the above term, yields

$$
\begin{aligned}
g(x)= & \int_{0}^{x} \sum_{k=0}^{\infty}(\lambda i)^{k}\left(\frac{x-t}{a k}\right)^{\frac{(\alpha+v+1) k+\alpha}{2}} J_{(\alpha+v+1) k+\alpha}(2 \sqrt{k a(x-t)}) f^{\prime}(t) d t \\
& +f(0) \sum_{k=0}^{\infty}(\lambda i)^{k}\left(\frac{x}{a k}\right)^{\frac{(\alpha+v+1) k+\alpha}{2}} J_{(\alpha+v+1) k+\alpha}(2 \sqrt{k a x}) .
\end{aligned}
$$

Finally, by taking the real and imaginary part of the above relation, we finally obtain the solutions of the system in the following forms

$$
\begin{aligned}
g_{1}(x) & =\sum_{k=0}^{\infty}\left(-\lambda^{2}\right)^{k} \int_{0}^{x}\left(\frac{x-t}{2 a k}\right)^{k(\alpha+v+1)+\frac{\alpha}{2}} J_{2 k(\alpha+v+1)+\alpha}(2 \sqrt{2 k a(x-t)}) f_{1}^{\prime}(t) d t \\
& -\sum_{k=0}^{\infty}(-1)^{k} \lambda^{(2 k+1)} \int_{0}^{x}\left(\frac{x-t}{(2 k+1) a}\right)^{\frac{(2 k+1)}{2}(\alpha+v+1)+\frac{\alpha}{2}} J_{(2 k+1)(\alpha+v+1)+\alpha}(2 \sqrt{a(2 k+1)(x-t)}) f_{2}^{\prime}(t) d t \\
& +f_{1}(0) \sum_{k=0}^{\infty}\left(-\lambda^{2}\right)^{k}\left(\frac{x}{2 a k}\right)^{k(\alpha+v+1)+\frac{\alpha}{2}} J_{2 k(\alpha+v+1)+\alpha}(2 \sqrt{2 k a x}) \\
& -f_{2}(0) \sum_{k=0}^{\infty}(-1)^{k} \lambda^{(2 k+1)}\left(\frac{x}{(2 k+1) a}\right)^{\frac{(2 k+1)}{2}(\alpha+v+1)+\frac{\alpha}{2}} J_{(2 k+1)(\alpha+v+1)+\alpha}(2 \sqrt{a(2 k+1) x}) .
\end{aligned}
$$

Similarly we get

$$
\begin{aligned}
g_{2}(x) & =\sum_{k=0}^{\infty}(-1)^{k} \lambda^{(2 k+1)} \int_{0}^{x}\left(\frac{x-t}{(2 k+1) a}\right)^{\frac{(2 k+1)}{2}(\alpha+v+1)+\frac{\alpha}{2}} J_{(2 k+1)(\alpha+v+1)+\alpha}(2 \sqrt{a(2 k+1)(x-t)}) f_{1}^{\prime}(t) d t \\
& +\sum_{k=0}^{\infty}\left(-\lambda^{2}\right)^{k} \int_{0}^{x}\left(\frac{x-t}{2 a k}\right)^{k(\alpha+v+1)+\frac{\alpha}{2}} J_{2 k(\alpha+v+1)+\alpha}(2 \sqrt{2 k a(x-t)}) f_{2}^{\prime}(t) d t \\
& +f_{1}(0) \sum_{k=0}^{\infty}(-1)^{k} \lambda^{(2 k+1)}\left(\frac{x}{(2 k+1) a}\right)^{\frac{(2 k+1)}{2}(\alpha+v+1)+\frac{\alpha}{2}} J_{(2 k+1)(\alpha+v+1)+\alpha}(2 \sqrt{a(2 k+1) x}) \\
& +f_{2}(0) \sum_{k=0}^{\infty}\left(-\lambda^{2}\right)^{k}\left(\frac{x}{2 a k}\right)^{k(\alpha+v+1)+\frac{\alpha}{2}} J_{2 k(\alpha+v+1)+\alpha}(2 \sqrt{2 k a x}) .
\end{aligned}
$$


Definition 1.1 Let $f(x, y)$ be a continuous function defined on the square $[0, \infty) \times[0, \infty)$, which is of exponential order, that is, for some $\lambda, \xi \in R^{+}$

$$
\sup _{x, y>0} \frac{|f(x, y)|}{e^{\lambda x+\xi y}}<\infty .
$$

Then the two dimensional Laplace transforms of $f(x, y)$ is defined as

$$
L_{2}\{f(x, y)\}=\int_{0}^{\infty} \int_{0}^{\infty} e^{-p x-q y} f(x, y) d x d y:=F(p, q) .
$$

If $L_{2}\{f(x, y)\}=F(p, q)$, then $L_{2}^{-1}\{F(p, q)\}$ is given by

$$
f(x, y)=\frac{1}{(2 \pi i)^{2}} \int_{\sigma-i \infty}^{\sigma+i \infty} \int_{c-i \infty}^{c+i \infty} e^{x p+y q} F(p, q) d p d q .
$$

Definition 1.2 The one-dimensional convolution of $f(x)$ and $g(x)$ is as

$$
(f * g)(x)=\int_{0}^{x} f(x-z) g(z) d z,
$$

Also, the two-dimensional convolution of $f(x, y)$ and $g(x, y)$ is given by

$$
(f * * g)(x, y)=\int_{0}^{x} \int_{0}^{y} f(x-\xi, y-\eta) g(\xi, \eta) d \eta d \xi
$$

\section{Evaluation of Integrals and Solution to Fractional P.D.E. By Means of the Two Dimensional Laplace Transform}

In this section, we evaluate the integrals that their evaluations are not an easy task. But, by means of two dimensional Laplace transform we can evaluate these integrals.

Lemma 2.1 The following integral relations hold true

$$
\text { a) } I=\frac{2}{\pi} \int_{0}^{\frac{\pi}{2}} \frac{1}{\tan \alpha} \operatorname{bei}\left(\frac{2}{\sqrt{\tan \alpha}}\right) d \alpha=J_{0}(2) \text {, }
$$

Where $\operatorname{bei}(z)=\operatorname{Im}\left(J_{0}\left(z e^{\frac{3 \pi i}{4}}\right)\right)$ and

$$
\text { b) } I=\frac{2}{\pi} \int_{0}^{\infty} \operatorname{bei}\left(\frac{2}{\sqrt{t}}\right) \frac{\cos t d t}{t}=\sum_{n=0}^{+\infty} \frac{(-1)^{n}}{(n !)^{3}} \text {. }
$$


Proof. (a) : By change of variable $t=\tan \alpha$, we get

$$
I=\frac{2}{\pi} \int_{0}^{\infty} \frac{1}{t\left(t^{2}+1\right)} \operatorname{bei}\left(\frac{2}{\sqrt{\mathrm{t}}}\right) d t
$$

Using the fact that

$$
L_{2}^{(p, q)}\left\{\operatorname{tbei}\left(2 \sqrt{\frac{x y}{t}}\right)\right\}=\frac{1}{p^{2} q^{2}+\frac{1}{t^{2}}}
$$

Let us assume that

$$
I(x, y)=\frac{2}{\pi} \int_{0}^{\infty} \frac{1}{t^{2}\left(t^{2}+1\right)}\left(\operatorname{tbei}\left(2 \sqrt{\frac{x y}{t}}\right)\right) d t
$$

Then, we get

$$
L_{2}^{(p, q)}\{I(x, y)\}=\frac{2}{\pi p^{2} q^{2}} \int_{0}^{\infty} \frac{d t}{\left(t^{2}+1\right)\left(t^{2}+\frac{1}{p^{2} q^{2}}\right)}=\frac{1}{1+p q}=\frac{1}{q} \frac{1}{p+\frac{1}{q}}
$$

The inverse two dimensional Laplace transform of the above relation yields

$$
I(x, y)=J_{0}(2 \sqrt{x y})
$$

Which concludes that?

$$
I=I(1,1)=J_{0}(2)
$$

Proof $(b)$ : Similarly, we obtain

$$
I=\sum_{n=0}^{\infty} \frac{(-1)^{n}}{(n !)^{3}}
$$

Theorem 2.1 (Sub Ballistic Fractional PDE) The following non - homogeneous partial fractional differential equations [17].

$$
{ }_{0}^{C} D_{x}^{\alpha} u(x, t)+{ }_{0}^{C} D_{t}^{\beta} u(x, t)=1: x, t>0
$$

Where $0<\alpha, \beta \leq 1$ with the boundary conditions $u(0, t)=u(x, 0)=0$, We get the following formal solution 


$$
u(x, t)=\sum_{n=0}^{\infty} \frac{(-1)^{n} x^{-n \alpha} t^{n+1}}{\Gamma(1-n \alpha) \Gamma(n+2)}
$$

Proof: At first, we assume that $0<\alpha \leq 1$ and $0<\beta<1$, then applying the two-dimensional Laplace transform term wise to P.D.E, yields

$$
p^{\alpha} U(p, q)+q^{\beta} U(p, q)=\frac{1}{p q}
$$

with $L_{2}\{u(x, t)\}=U(p, q)$. Therefore

$$
U(p, q)=\frac{1}{p q\left(p^{\alpha}+q^{\beta}\right)}=\frac{1}{p^{\alpha+1} q\left(1+\frac{q^{\beta}}{p^{\alpha}}\right)}=\sum_{n=0}^{\infty} \frac{(-1)^{n} q^{n \beta-1}}{p^{(n+1) \alpha+1}} .
$$

The inverse two-dimensional Laplace transforms yields

$$
u(x, t)=\sum_{n=0}^{\infty} \frac{(-1)^{n} x^{(n+1) \alpha} t^{-n \beta}}{\Gamma(n \alpha+\alpha+1) \Gamma(1-n \beta)} .
$$

For the special case $\alpha=\beta=0.5$, we have

$$
u(x, t)=\frac{2}{\sqrt{\pi}}\left(\sqrt{x}-\frac{x}{\sqrt{t}+\sqrt{x+t}}\right)
$$

And the figure is shown as follows.

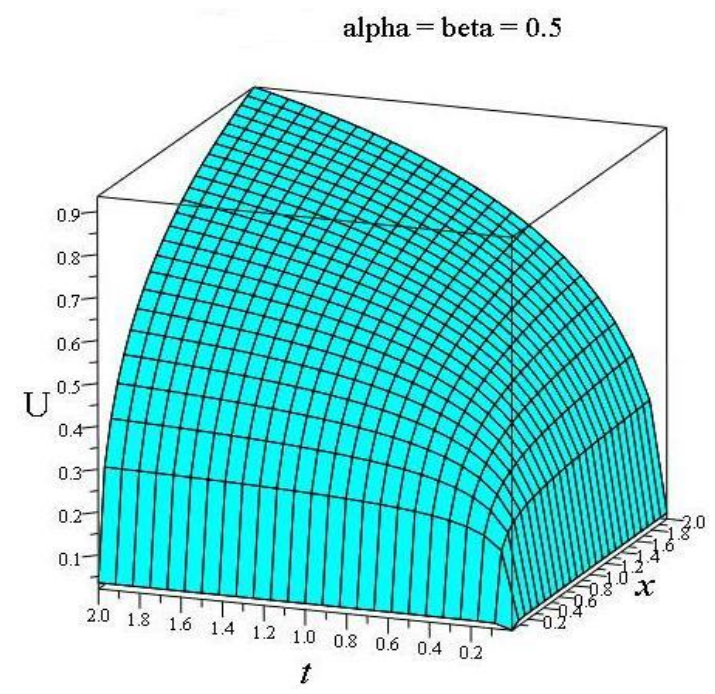


Note that when $0<\alpha<1$ and $\beta=1$, by using the two-dimensional Laplace transform, we may calculate $U(p, q)$ as follows,

$$
U(p, q)=\frac{1}{p q\left(p^{\alpha}+q\right)}=\frac{1}{p q^{2}\left(1+\frac{p^{\alpha}}{q}\right)}=\sum_{n=0}^{\infty} \frac{(-1)^{n} p^{n \alpha-1}}{q^{n+2}},
$$

So that

$$
u(x, t)=\sum_{n=0}^{\infty} \frac{(-1)^{n} x^{-n \alpha} t^{n+1}}{\Gamma(1-n \alpha) \Gamma(n+2)} .
$$

For $\alpha=1$ and $\beta=0.5$, we have the following

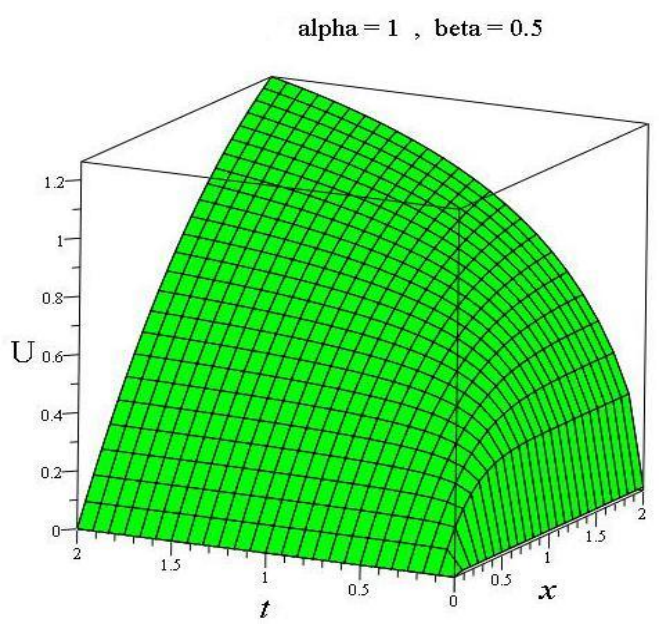

In special case, let us take $\alpha=\beta=1$, the Laplace transform with respect to $t$ gives

$$
U_{x}(x, q)+q U(x, q)=\frac{1}{q},
$$

Therefore

$$
U(x, q)=\frac{1}{q^{2}}\left(1-e^{-q x}\right) .
$$

At this point, the inverses Laplace transform yields

$$
u(x, t)=t-(t-x) H(t-x)=\left\{\begin{array}{ll}
t & : x>t \\
x & : x<t
\end{array} .\right.
$$




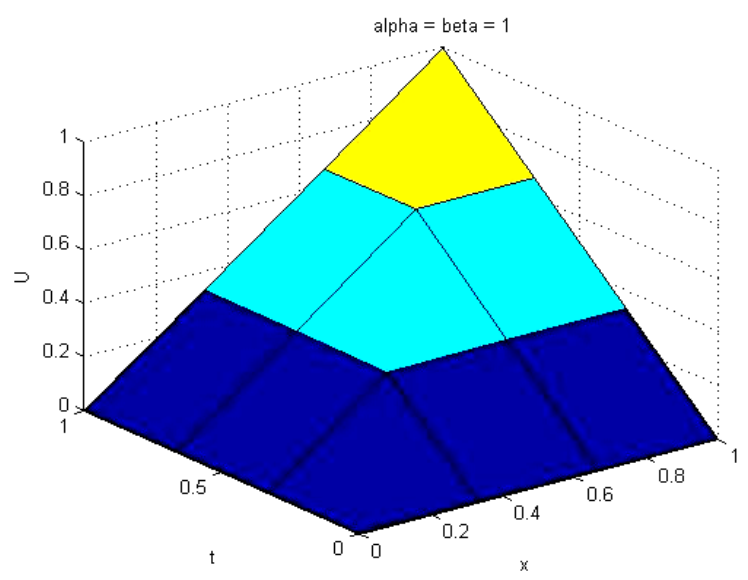

\section{Solving System of Time Fractional Heat Equations}

In this section, the authors considered certain homogeneous system of time fractional heat equations which is a generalization to the problem of thermal effects on fluid flow and hydraulic fracturing from well bores and cavities in low permeability formations [12]. In this work, only the Laplace transformation is considered as it is easily understood and being popular among engineers and scientists.

Problem3.1 Solving the homogeneous systems of time fractional heat equations [12]

$$
\begin{gathered}
{ }_{0}^{C} D_{t}^{\alpha} u=k^{2} \frac{\partial^{2} u}{\partial x^{2}} \\
{ }_{0}^{C} D_{t}^{\alpha} v=k^{2} \frac{\partial^{2} v}{\partial x^{2}}+\lambda^{2}{ }_{0}^{C} D_{t}^{\alpha} u
\end{gathered}
$$

Where $0<\alpha \leq 1,0<x<\infty, t>0$ with the boundary conditions,

$$
u(0, t)=1, \lim _{x \rightarrow+\infty}|u(x, t)|<\infty, v_{x}(0, t)=-1, \lim _{x \rightarrow+\infty}|v(x, t)|<\infty
$$

And the initial conditions are $u(x, 0)=v(x, 0)=0$.

Solution: Application of the Laplace transform to the first equation and the initial conditions, yields

$$
s^{\alpha} U(x, s)=k^{2} U_{x x}(x, s) .
$$

Now, the boundary conditions give

$$
U(x, s)=\frac{1}{s} e^{-\frac{s^{\frac{\alpha}{2}}}{k} x}=\sum_{n=0}^{\infty} \frac{\left(-\frac{x}{k}\right)^{n}}{n !} \frac{1}{s^{1-\frac{n \alpha}{2}}}
$$


That leads to

$$
u(x, t)=\sum_{n=0}^{\infty} \frac{\left(-\frac{x}{k}\right)^{n}}{n !} \frac{t^{-\frac{n \alpha}{2}}}{\Gamma\left(1-\frac{n \alpha}{2}\right)} .
$$

The special case $\alpha=1$ gives

$$
u(x, t)=\operatorname{erfc}\left(\frac{x}{2 k \sqrt{t}}\right) .
$$

Similarly, applying the Laplace transform of the second equation and using the initial conditions, we arrive at

$$
V_{x x}(x, s)-s^{\alpha} V(x, s)=-s^{\alpha-1} e^{-\frac{s^{\frac{\alpha}{2}}}{k} x} .
$$

Using the boundary conditions, solution of the above differential equation is as

$$
V(x, s)=\left(\frac{\lambda^{2}}{2 s}+\frac{k}{s^{\frac{\alpha}{2}+1}}+\frac{x \lambda^{2}}{2 k s^{1-\frac{\alpha}{2}}}\right) e^{-\frac{s^{\frac{\alpha}{2}}}{k} x} \text {. }
$$

The Laplace transform inversion formula leads to the following formal solution

$$
v(x, t)=\sum_{n=0}^{\infty} \frac{\left(-\frac{x}{k}\right)^{n}}{n !}\left(\frac{\lambda^{2}}{2} \frac{t^{-\frac{n \alpha}{2}}}{\Gamma\left(1-\frac{n \alpha}{2}\right)}+k \frac{t^{\frac{\alpha}{2}-\frac{n \alpha}{2}}}{\Gamma\left(1+\frac{\alpha}{2}-\frac{n \alpha}{2}\right)}+\frac{\lambda^{2} x}{2 k} \frac{t^{-\frac{\alpha}{2}-\frac{n \alpha}{2}}}{\Gamma\left(1-\frac{\alpha}{2}-\frac{n \alpha}{2}\right)}\right)
$$

For the special case $\alpha=1$, we obtain

$$
v(x, t)=\left(\frac{\lambda^{2}}{2}-x\right) \operatorname{erfc}\left(\frac{x}{2 k \sqrt{t}}\right)+\left(2 k \sqrt{\frac{t}{\pi}}+\frac{\lambda^{2} x}{2 k \sqrt{\pi t}}\right) e^{-\frac{x^{2}}{4 k^{2} t}} .
$$

We have shown the solutions in the following figures for $\alpha=1,0.5$ and $\lambda=k=1$ 

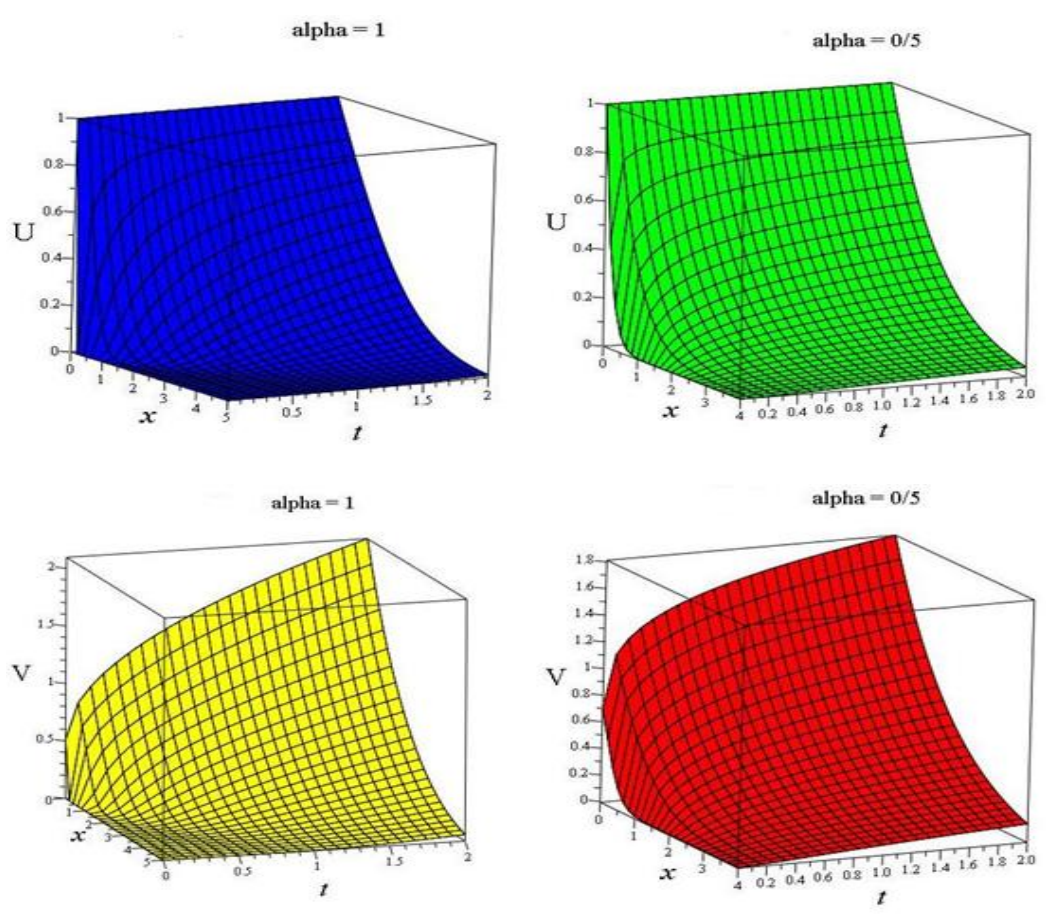

\section{Effect of a Uniform Overburden on the Passage of a Thermal Wave}

In this section, the authors considered certain homogeneous time fractional heat equations which are a generalization of the problem of the effect of a uniform overburden on the passage of a thermal wave and the temperatures in the underlying rock studied by D.S. Parasnis [16].

Problem 4.1 Let us find the fractional heat flow in a two-layer earth [12]

$$
\begin{gathered}
{ }_{0}^{C} D_{t}^{\alpha} u_{1}(x, t)=a_{1} \frac{\partial^{2} u_{1}(x, t)}{\partial x^{2}}: 0 \leq x<h, t>0 \\
{ }_{0}^{C} D_{t}^{\alpha} u_{2}(x, t)=a_{2} \frac{\partial^{2} u_{2}(x, t)}{\partial x^{2}}: h \leq x, t>0
\end{gathered}
$$

Where $0<\alpha \leq 1$ and $a_{i}$ denotes the diffusivity of the layer, $u_{i}$ denotes the temperature and $x$ denotes the distance down from the surface. At the earth's surface, there is a diurnal cycle $u_{1}(0, t)=T_{0} \sin \omega t$, while at the interface between the two layers,

$$
u_{1}(h, t)=u_{2}(h, t), k_{1} \frac{\partial u_{1}(h, t)}{\partial x}=k_{2} \frac{\partial u_{2}(h, t)}{\partial x}
$$

Where $k_{i}$ denotes the thermal conductivity. We also require that $\lim _{x \rightarrow \infty}\left|u_{2}(x, t)\right|<\infty$. The initial conditions $\operatorname{are} u_{1}(x, 0)=u_{2}(x, 0)=0$. 
Solution: Taking the Laplace transform of two equations, we get

$$
\frac{d^{2} U_{i}(x, s)}{d x^{2}}-\frac{s^{\alpha}}{a_{i}} U_{i}(x, s)=0 \quad(i=1,2)
$$

Therefore, using the initial and boundary conditions, we get the following

$$
\left.U_{1}(x, s)=\frac{T_{0} w}{s^{2}+w^{2}} \exp \left(x \sqrt{\frac{s^{\alpha}}{a_{1}}}\right) \frac{\beta \exp \left(-2 h \sqrt{\frac{s^{\alpha}}{a_{1}}}\right)}{1+\beta \exp \left(-2 h \sqrt{\frac{s^{\alpha}}{a_{1}}}\right)}+\frac{T_{0} w}{s^{2}+w^{2}} \frac{\exp \left(-x \sqrt{\frac{s^{\alpha}}{a_{1}}}\right)}{1+\beta \exp \left(-2 h \sqrt{\frac{s^{\alpha}}{a_{1}}}\right.}\right)
$$

And

$$
U_{2}(x, s)=\frac{T_{0} w(1+\beta)}{s^{2}+w^{2}} \frac{\exp \left[h\left(\sqrt{\frac{s^{\alpha}}{a_{2}}}-\sqrt{\frac{s^{\alpha}}{a_{1}}}\right)-x \sqrt{\frac{s^{\alpha}}{a_{2}}}\right]}{1+\beta \exp \left(-2 h \sqrt{\frac{s^{\alpha}}{a_{1}}}\right)}
$$

Where $\beta=\frac{k_{1} \sqrt{a_{2}}-k_{2} \sqrt{a_{1}}}{k_{1} \sqrt{a_{2}}+k_{2} \sqrt{a_{1}}}$. To invert $U_{1}$ and $U_{2}$, we observe that

$$
\frac{1}{1+\beta \exp \left(-2 h \sqrt{\frac{s^{\alpha}}{a_{1}}}\right)}=\sum_{n=0}^{\infty}(-1)^{n} \beta^{n} \exp \left(-2 n h \sqrt{\frac{s^{\alpha}}{a_{1}}}\right),
$$

if $\left|\beta \exp \left(-2 h \sqrt{\frac{s^{\alpha}}{a_{1}}}\right)\right|<1$. Then we get the following relations

$$
\begin{aligned}
U_{1}(x, s) & =\frac{T_{0} w}{s^{2}+w^{2}} \sum_{n=0}^{\infty}(-1)^{n} \beta^{n+1} \exp \left[-(2 n h+2 h-x) \sqrt{\frac{s^{\alpha}}{a_{1}}}\right] \\
& +\frac{T_{0} w}{s^{2}+w^{2}} \sum_{n=0}^{\infty}(-\beta)^{n} \exp \left[-(2 n h+x) \sqrt{\frac{s^{\alpha}}{a_{1}}}\right]
\end{aligned}
$$

And

$$
U_{2}(x, s)=\frac{T_{0} w(1+\beta)}{s^{2}+w^{2}} \sum_{n=0}^{\infty}(-\beta)^{n} \exp \left\{-\left[(2 n+1) h+(h-x) \sqrt{\frac{a_{1}}{a_{2}}}\right] \sqrt{\frac{s^{\alpha}}{a_{1}}}\right\}
$$


We can show (by using convolution) that the inverse of

$$
F(z, s)=\left(\frac{w}{s^{2}+w^{2}}\right) \exp \left(-z \sqrt{s^{\alpha}}\right)
$$

Is as

$$
f(z, t)=\frac{1}{t} \int_{0}^{t} \sin (\omega(t-\tau)) W\left(-\frac{\alpha}{2}, 0 ;-\frac{z}{\tau^{\frac{\alpha}{2}}}\right) d \tau .
$$

Where $W(.,$.$) stands for the Wright function. The final solutions are$

$$
u_{1}(x, t)=T_{0} \sum_{n=0}^{\infty}(-1)^{n} \beta^{n+1} f\left(\frac{2 n h+2 h-x}{\sqrt{a_{1}}}, t\right)+T_{0} \sum_{n=0}^{\infty}(-\beta)^{n} f\left(\frac{2 n h+x}{\sqrt{a_{1}}}, t\right)
$$

And

$$
u_{2}(x, t)=T_{0}(1+\beta) \sum_{n=0}^{\infty}(-\beta)^{n} f\left(\frac{(2 n+1) h+(h-x) \sqrt{\frac{a_{1}}{a_{2}}}}{\sqrt{a_{1}}}, t\right) .
$$

\section{Conclusion}

In this paper, analytic solution of the space - time fractional sub - ballistic and heat equations are derived using the integral transform method. The authors implemented two dimensional Laplace transform to solve non -homogeneous sub ballistic fractional PDE and homogeneous systems of time fractional heat equations. Illustrative examples are given.

\section{References}

[1] A. Aghili; M.R. Masomi. Solution to Time Fractional Partial Differential Equations and Dynamical Systems via Integral Transforms. Journal of Interdisciplinary Mathematics, Vol 14, No 5 \& 6, pp 545-560, 2011.

[2] A. Aghili; M.R. Masomi. The Double Post-Widder Inversion Formula for Two Dimensional Laplace Transform and Stieltjes Transform with Applications. Bulletin of Pure and Applied Sciences, Volume 30 E (Math \& Stat.) Issue (No.2), 191-204, and 2011.

[3] A. Aghili; M.R. Masomi. Solution to Time Fractional Partial Differential Equations via Joint Laplace-Fourier Transforms. Journal of Interdisciplinary Mathematics, Vol 15, No 2 \& 3, pp 121-135, 2012.

[4] A. Aghili; M.R. Masomi. Transform Method for Solving Time-Fractional Order Systems of Nonlinear Differential and Difference Equations. Antarctica J. Math., 10(3), 237-251, 2013.

[5] A. Aghili; M.R. Masomi. Integral Transform Method for Solving Different F.S.I.Es and P.F.D.Es. Konuralp Journal of Mathematics, Volume 2, No. 1 pp. 45-62, 2014.

[6] A. Aghili; M.R. Masomi. Integral Transform Method for Solving Time Fractional Systems and Fractional Heat Equation. Boletim da Sociedade Paranaense de Matemática, Vol 32, No 1, pp 305-322, 2014. 
[7] A. Aghili; M.R. Masomi. Solution to Partial Fractional Differential Equations With Moving Boundaries in Two Dimensions. International Journal of Innovation in Science and Mathematics, Vol 2, 26-30, 2014.

[8] A. Aghili; M.R. Masomi. Nonhomogeneous Generalized Multi-Term Fractional Heat Propagation and Fractional Diffusion-Convection Equation in Three-Dimensional Space. New Trends in Mathematical Sciences, Vol. 2, No. 2, 2014, p106-116, 2014.

[9] A. Aghili; M.R. Masomi. Perturbation Method for Linear and Non-Linear Fractional Order Systems and Integral Representation For Evaluation of Integrals. Boletim da Sociedade Paranaense de Matemática, Vol 33 1, 1-22, 2015.

[10] A.M. Cohen. Numerical Methods for Laplace Transform Inversion. Springer, 2007.

[11] V.A. Ditkin; A.P. Prudnikov. Calcul Operationnelle. Mir publisher. 1979.

[12] D.G. Duffy. Transform Methods for Solving Partial Differential. Chapman \& Hall/CRC, 2004.

[13] Y. Brychkov; A.P. Glaeske, A.P. Prudnikov, Vu Kim Tuan. Multidimensional Integral Transformations. Gordon and Breach Science Publishers, 1992.

[14] A.A. Kilbas; H.M. Srivastava; J.J. Trujillo. Theory and Applications of Fractional Differential Equations. Elsevier, 2006.

[15] I. Podlubny. Fractional Differential Equations. Academic Press, 1999.

[16] D.S. Parasnis. Effect of a Uniform Overburden on the Passage of a Thermal Wave and the Temperatures in the Underlying Rock. Geophys. J.R.Astr.Soc 46,189 -192, 1976.

[17] V.V.Uchaikin. Fractional Derivatives for Physicists and Engineers Vol.1 Springer 2012.

\footnotetext{
*Corresponding author.

E-mail address: armanaghili@yahoo.com/ masomirasool@ yahoo.com
} 